\title{
A FPGA Approach in a Motorised Linear Stage Remote Controlled Experiment
}

\author{
http://dx.doi.org/10.3991/ijoe.v9iS3.2543 \\ S.D. Gadzhanov, A. Nafalski and Z. Nedic \\ University of South Australia, Mawson Lakes, Australia
}

\begin{abstract}
In recent years, an advanced motion control software for rapid development has been introduced by National Instruments, accompanied by innovative and improved FPGA-based hardware platforms. Compared to the well-known standard NI DAQ PCI/USB board solutions, this new approach offers robust stability in a deterministic real-time environment combined with the highest possible performance and re-configurability of the FPGA core. The NI Compact RIO (cRIO) Real Time Controller utilises two distinctive interface modes of functionality: Scan and FPGA modes. This paper presents an application of a motion control flexible workbench based on the FPGA module, and analyses the advantages and disadvantages in comparison to another approach - the LabVIEW NI SoftMotion module run in scan interface mode. The workbench replicates real industrial applications and is very useful for experimentation with Brushless DC/ Permanent Magnet Synchronous motors and drives, and feedback devices.
\end{abstract}

Index Terms-Brushless DC motor; LabVIEW; motion control; RT target; FPGA target; SoftMotion.

\section{INTRODUCTION}

Remote laboratories for motion control, part of the growing field of computerised laboratories, emerge as a valuable tool for performing real time experiments with usually expensive or unique laboratory equipment in a safe way. The development and constant improvement of software applications such as National Instruments (NI) LabVIEW and Mathworks MATLAB allow easy implementation of control algorithms and creation of user friendly interfaces with virtual instruments (VI). The use of embedded systems with integrated Field Programmable Gate Arrays (FPGAs) increases the robustness, reliability and determinism of the control applications [1-4].

While a motion control application with a NI PCI/USB PC-based data-acquisition board offers an advanced functionality and high sampling rates, it lacks the rigidness and the high control loop rates achievable with the embedded FPGA system.

The NI cRIO-9076 embedded controller [5] represents a highly reliable and flexible combination of robust and deterministic real-time operating system, FPGA core and dedicated LabVIEW software modules for rapid development of control and monitoring applications. The controller chassis and the communication interfaces provide an access to a wide range of DAQ modules and devises in conjunction with the targeted application.

The National Instrument cRIO C-series Drive Interface modules are an integrated hardware environment for various single axis motion control applications. The NI 9516 C-series module [6] is a precise motion control servo drive interface for torque/position control, including separate microprocessor with integrated functionality for spline interpolation, PID and PID feed-forward control

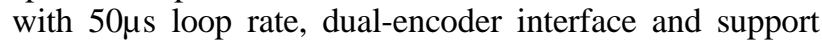
for custom digital inputs and outputs, limit switches.

The NI SoftMotion module [7] is a valuable addition to the LabVIEW graphical development environment. Users benefit from the high-level application programming interface (API), providing rapid means for development of custom-oriented motion control applications in Scan or FPGA mode. The key futures could be determined as:

- High-level visual axes customisation with the ability to define additional inputs and outputs, which is NI C-series modules oriented;

- Integrated support for End-limit switches and Home switch, Brakes, Gearing and Camming;

- Integrated functionality for trajectory generation and spline interpolation;

- Dedicated Functional Blocks for movements, axis referencing, status monitoring, etc.;

- Low level programming with Property, Invoke and Axis Interface nodes for more complex system definition and control.

The NI LabVIEW FPGA module [8] is other versatile LabVIEW graphical development environment. Its main feature is the compilation and HDL code implementation of the developed LabVIEW VIs into the FPGA hardware target. Its other functionality can be summarised as:

- High control loop rates;

- Determinism and parallelism in the execution of multiple program routines;

- Development and simulation of the application software on a host PC computer;

- Development of custom control logic and timing, and communication protocols.

This article describes the application of the NI FPGA module in an experimental remote laboratory motion control workbench (presented in [9]) consisting of: Linear stage with an optical linear scale, BLDC motor with sinusoidal Back-EMF, High-performance FPGA-based motion controller MC73110 [10] with Field-Oriented Control (FOC) and NI cRIO embedded system.

Firstly, the hardware set-up of the workbench will be explained in details. Then a motion control application of the NI FPGA module will be presented. Its performance and functionality are compared with another two solutions based on the combinations of the NI SoftMotion module/cRIO controller and the LabVIEW for Windows/ NI USB data-acquisition board. 


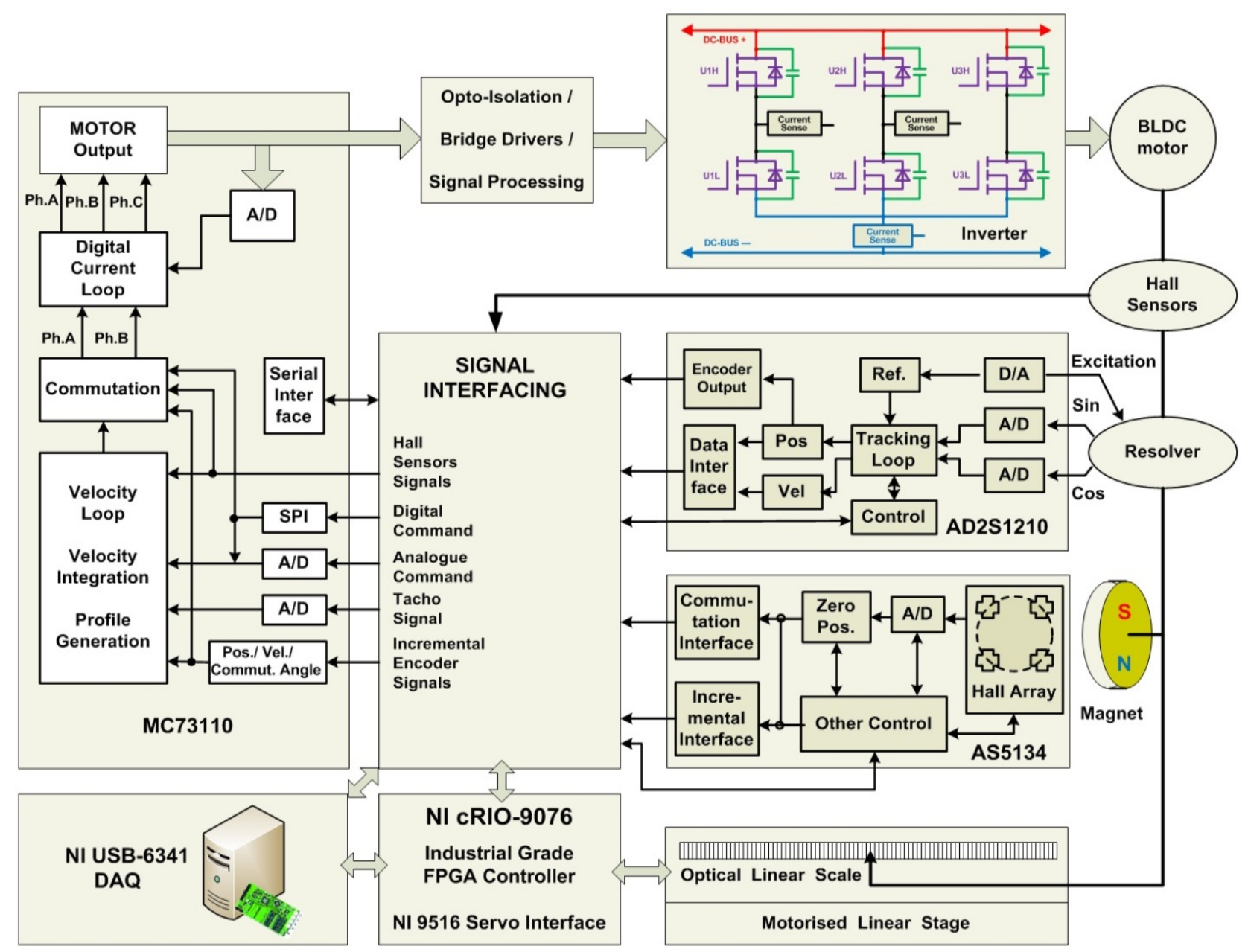

Figure 1. System Block Diagram of the Experimental Workbench

\section{HARDWARE SET-UP OF THE MOTION CONTROL WORKBENCH}

The developed motion control workbench replicates a motorised axis, widely used in industrial CNC machines and robots. This chapter describes the hardware structure, the functional block diagram of interaction between the hardware/software modules and the high-performance motion controller MC73110; its initialisation and diagnostics screen.

\section{A. Hardware Structure:}

A system block diagram which demonstrates the interaction between the main electro-mechanical modules is depicted in in Figure 1 and is described in more details in [9]. The platform is a mechanical system with three control loops: current/torque, velocity and position. The following components have been used to utilise its functionality:

12 inch linear stage - a type of linear actuator where the rotary motion is translated into a linear motion. It consists of a base and a moving table (platform) which motion is restricted in one dimension by linear slides. The moving table is attached to a ball screw with an antibacklash lead-screw assembly. The ball screw is coupled with the motor by means of a helical flexible coupling.

BLDC motor - a four-pole motor with very close to sinusoidal Back-EMF, nominal voltage $36 \mathrm{~V}$, rated speed 4000rpm, rated torque $0.4 \mathrm{Nm}$, torque constant 0.063 Nm/A and standard NEMA 23 flange mounting.

MC73110 [10] - an FPGA based, high-performance motion control IC, which incorporates all the needed internal blocks to work as a stand-alone servo controller. In addition, online programming is available by means of RS232 or Synchronous Serial Input (SPI) interfaces. It utilises a trapezoidal and sinusoidal types of control and is also capable of Field Oriented Control. Its control loops are depicted in Figure 2: Current (motor phases A/B), Commutation (Hall-effect sensors), Velocity and Velocity Integrator (DC tacho-generator and incremental encoder signals). It drives an inverter comprising a full HEXFET power transistor three-phase bridge.

Precision optical linear scale - a $320 \mathrm{~mm}$ precision enclosed optical linear scale with incremental encoder interface has been used for a linear position feedback.

Rotor Excited Resolver - a frameless hollow-shaft rotary electrical transformer has been used for an angular position feedback of the motor rotor. It has an input (primary) winding which is excited with a sinusoidal signal. The two output (secondary) windings are displaced by 90 degrees and therefore produce sinusoidal and cosinusoidal signals.

AD2S1210 [11] - in order to evaluate the output signals of the resolver, a complete 10-bit to 16-bit resolution tracking resolver-to-digital converter (RDC) has been used. It employs an integrated programmable sinusoidal oscillator that provides sinusoidal wave excitation for 


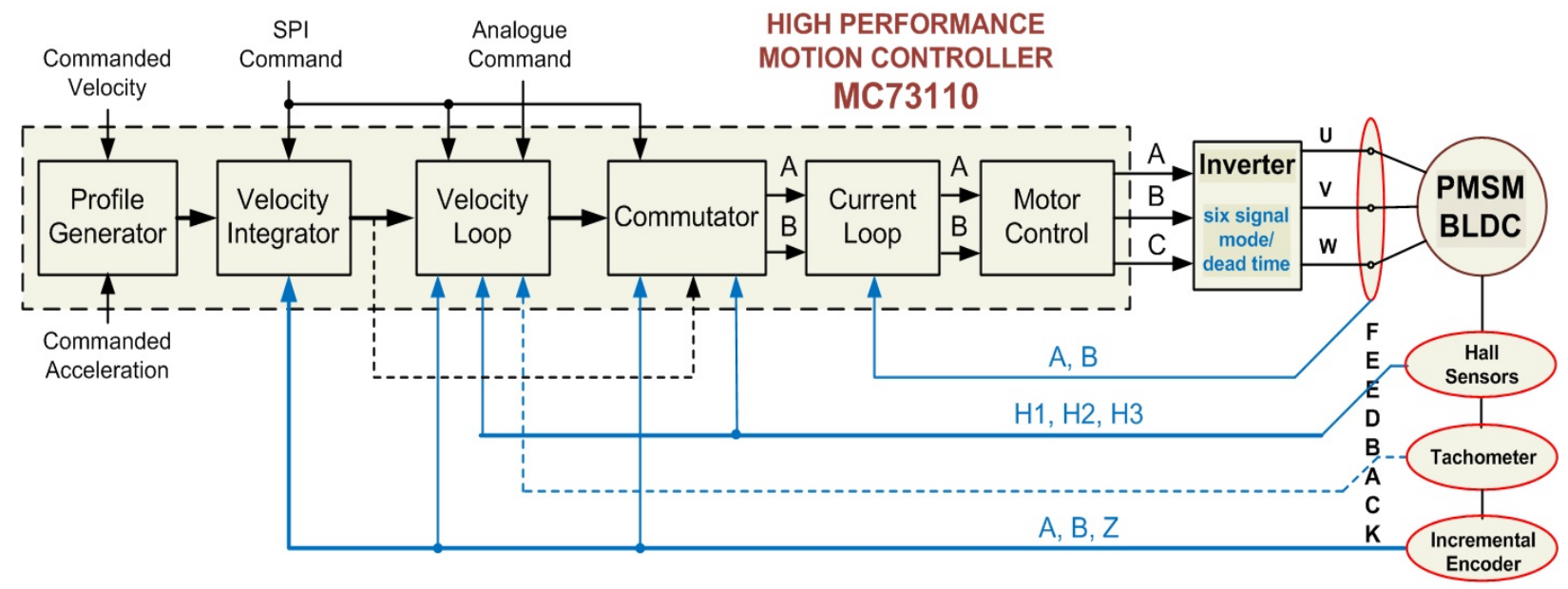

Figure 2. Control Loops of the PMD MC73110 High-Performance Motion Controller /adapted and modified from [10] /

resolvers. It can emulate an incremental quadrature encoder, and output data for the angular position or velocity.

NI USB-6341 [12] - an advanced high-performance DAQ, which provides new timing and synchronisation technologies, an enhanced data streaming over the USB interface and new NI-DAQmx LabVIEW drivers and virtual instruments. It utilises:

- Quadrature encoder specialised inputs with extended functionality;

- 32-bit Counter Inputs/Outputs with extended triggering functionality and $100 \mathrm{MHz}$ maximum source frequency;

- High speed $500 \mathrm{kS} / \mathrm{s}$ sampling with 16-bit resolution of the analogue inputs;

- High speed $900 \mathrm{kS} / \mathrm{s}$ sampling with 16-bit resolution of the analogue outputs;

- High speed $1 \mathrm{MHz}$ hardware and software timing of the bidirectional digital inputs/outputs and watchdog timer.

The main application of the board is high speed signal monitoring and control, system identification and model validation of the controlled dynamical system.

NI cRIO-9076 [5] - a stand-alone flexible embedded architecture with real-time operating system capable of execution of deterministic LabVIEW applications and:

- $\quad 400 \mathrm{MHz}$ PowerPC industrial grade processor;

- 256MB RAM and 512MB Nonvolatile memory;

- VxWorks robust real-time OS for control, datalogging and analysis; deterministic behaviour;

- National Instruments Scan engine;

- Xilinx Spartan-6 LX45 FPGA with 43661 logic sells, 58 multipliers and 2088Kb RAM;

- Highly reconfigurable cassis, accommodating up to four modules, Ethernet, USB and RS232.

NI 9516 C-series module [6] - a precise motion control servo drive interface with:

- Dedicated processor for spline interpolation, PID and PIDff control and $50 \mu \mathrm{s}(20 \mathrm{kHz})$ loop rate;

- Dual incremental encoder interface for position and velocity feedback;
- Customised support for digital inputs and outputs, including end-limit and home switches;

- $\quad$ Position capture and position compare I/Os.

\section{B. Functional Block Diagram of the Motion Control Workbench}

The host LabVIEW application is running on the PC, exchanging variables with the nondeterministic loop (a low-priority application) on the real time target (the NI cRIO system) through the network as shown in Figure 3. A second timed loop, which is deterministic, is running in real time the high-priority application and is synchronised with the NI Scan engine. This application is communicating with the NI 9516 servo interface module, requiring data from the experiment and writing it into shared variables, using a Real-Time FIFO. The acquired data is sent from the deterministic loop to the nondeterministic loop by means of Shared Variable Nodes. Then the non-deterministic loop is using NetworkPublished Shared Variables to send this data back to the host application for analysis and plotting.

It is important to mention that the NI cRIO-9076 highperformance controller allows two different kinds of interface programming modes to be used in conjunction with the real-time target:

- Scan Interface - a high-level programming interface, running a hardware-timed scan loop on the FPGA. It allows synchronous update rates of up to $1 \mathrm{kHz}$. However, the advantage of using NI C-series modules is the combination of highspeed performance (since these modules contain the high-speed circuitry) and the convenience of the high-level programming, thus reducing dramatically the development time;

- FPGA Interface - the highest possible performance, flexibility and reliability could be achieved in this mode; drawback sometimes is the very long compilation time; some applications could be developed first in scan mode and tested, and then redeveloped in FPGA mode. A hybrid programming mode is also possible, which gives developers additional advantages.

Apart from the standard PID/PIVff control algorithms which have been already implemented in the NI 9516 module, more complex and advanced algorithms (e.g. 


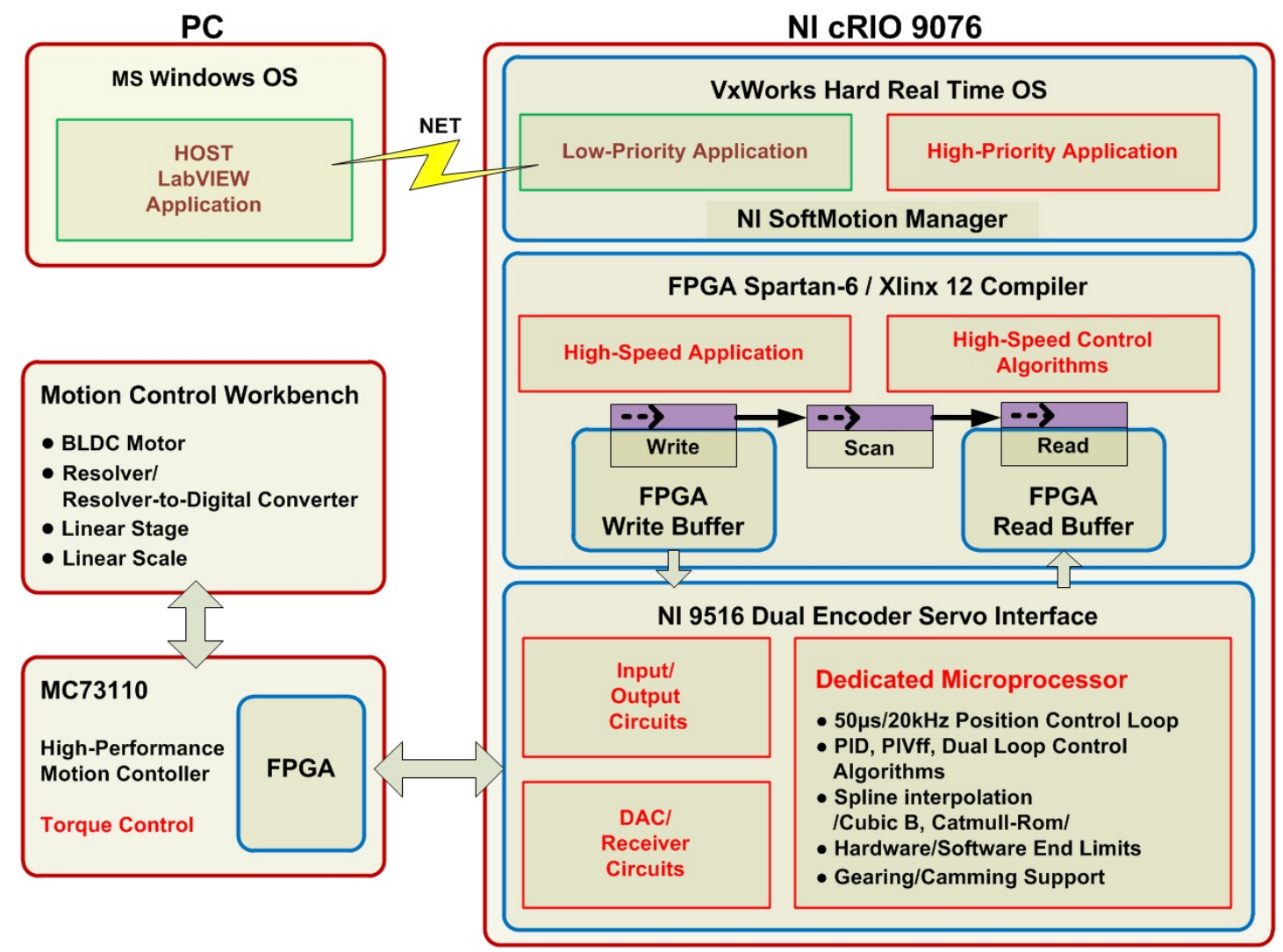

Figure 3. Functional Block Diagram of the Motion Control Workbench

Fuzzy-logic, LQR and H-infinity) can be developed and executed on the FPGA target for maximum performance and reliability.

\section{MC73110 Servo System Diagnostic Window}

For the purpose of initialisation and diagnostics of the MC73110 motion controller, more than 130 intelligent profile commands have been implemented in a new LabVIEW Virtual Instrument. It allows the controller to be easier set-up and tuned, the MC73110 control loops to be adjusted and important diagnostic information to be read during the control experiment.

The LabVIEW diagnostic screen has been divided into various diagnostic windows, according to their purpose and use:

- $\quad$ Activity Status and Limits - measured DC bus voltage and board temperature, and limits, etc.;

- Signal Sensing - a visual indication is shown for Hall-sensors phases, incremental encoder signals, amplifier and PWM output activation, emergency stop and the signal sense mask;

- Motor Status - provides information about the motor control: type of the loop, engaged feedback loops, velocity command source, type of commutation and phase correction - Figure 4;

- Serial Communication - provides complete information about the MC73110 serial communication via the RS232 and SPI ports;

- $\quad$ Events Status - provides information about faults related to motion or programmable amplifier, EEPROM/Flash processing; occurrence of encoder position wrapping and position capture;
- PWM Adjustment of the inverter bridge - bridge control mode, PWM frequency, duty cycle and dead time, sampling time, etc.;

- PID Parameters - for current, velocity and velocity-integrator loops;

- Condition Mask - provides information about the masks applied to the status registers for amplifier error, amplifier disable and PWM disable;

- Analogue Signals Status - shows the captured analogue voltage for the analogue command, tachometer and motor currents;

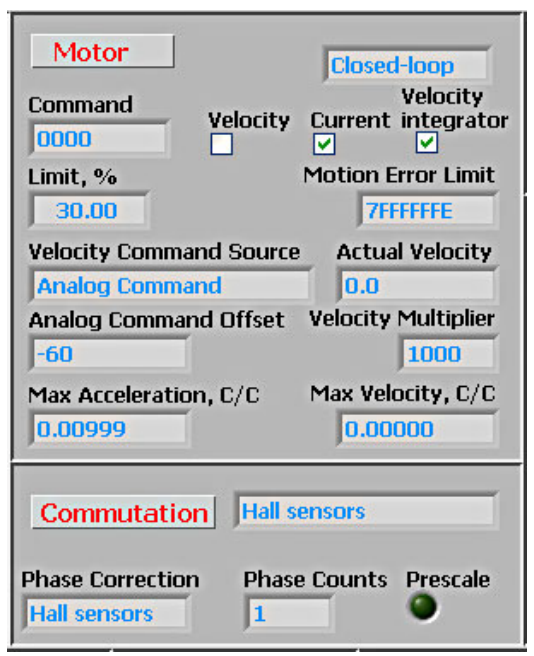

Figure 4. MC73110 Motor and Commutation Status Window 
- Other information for command status and various errors, related to currents, velocity, velocity integrator, etc.

- A Control Application of NI FPGA Module

The NI LabVIEW FPGA module provides a variety of tools and virtual instruments which allow programming, testing and implementation of a whole motion control system. This subchapter describes the host and the FPGA target applications, the tools for data exchange and the methods to accomplish the desired results.

\section{Tools for data exchange with the FPGA Target}

In order to achieve a high speed control and signal acquisition, the following tools and methods for data exchange between the host application and the FPGA target have been utilised:

\section{1) FPGA Memory Allocation}

The usage of the available on-board FPGA memory has two advantages - it avoids the reallocation of large areas of FPGA gates for arrays and increases the functionality, flexibility and interchangeability of the developed FPGA software.

In this application, the FPGA memory has been used for generation of custom arbitrary waveforms - the excitation signals for control experimentations. It contains 10000 points of the waveform, presented as 16-bit integer elements and containing the time stamps and moment amplitudes of the excitation signals:

- $\quad$ Square pulse signal;

- $\quad$ Pseudo-Random Binary Sequence (PRBS);

- $\quad$ Swept sine signal.

An additional VI has been developed for generation and storage of waveform data in dedicated arrays. These arrays are used by the host application to initialise the FPGA Block Memory domains with the same number of elements. Using the Read Memory method, the FPGA target application accesses the data for the chosen excitation signal and outputs it to the PID regulator.

\section{2) Signal Acquisition - FIFO tables}

One of the advantages of the NI cRIO 9076 controller are the five Direct Memory Access (DMA) channels, which provide data streaming from the FPGA target to the host application by means of FIFO tables. The latter have been used for high-speed signal acquisition of:

- $\quad$ Excitation voltage, (V);

- Motor torque (expressed in command voltage);

- $\quad$ Linear scale position, (cm);

- Angular encoder (resolver) position, (cm);

- $\quad$ FPGA loop period, ( $\mu \mathrm{s})$.

These FIFO tables have been created separately in the LabVIEW project and initialised with 10000 16-bit integer elements.

3) FPGA Target - NI 9516 module Data Exchange

Every FPGA cycle a configuration data is written into the NI 9516 module and consecutively - a status data is read from it. To accomplish this, three invoke methods are used - Figure 5:

- Write method - sends a set of NI 9516 module specific parameters to the FPGA Write Buffer. In the prototype, it enables the axis and the external servo controller (MC73110), and outputs the analogue command voltage;

- Scan method - moves the data from the FPGA Write Buffer to the NI 9516 module and at the same time reads the acquired data from the module and writes it into the FPGA Read Buffer within $40 \mu$ s period assured period of time;

- $\quad$ Read method - reads the required parameters from the FPGA Read Buffer and empties it. In the prototype, it delivers position information from

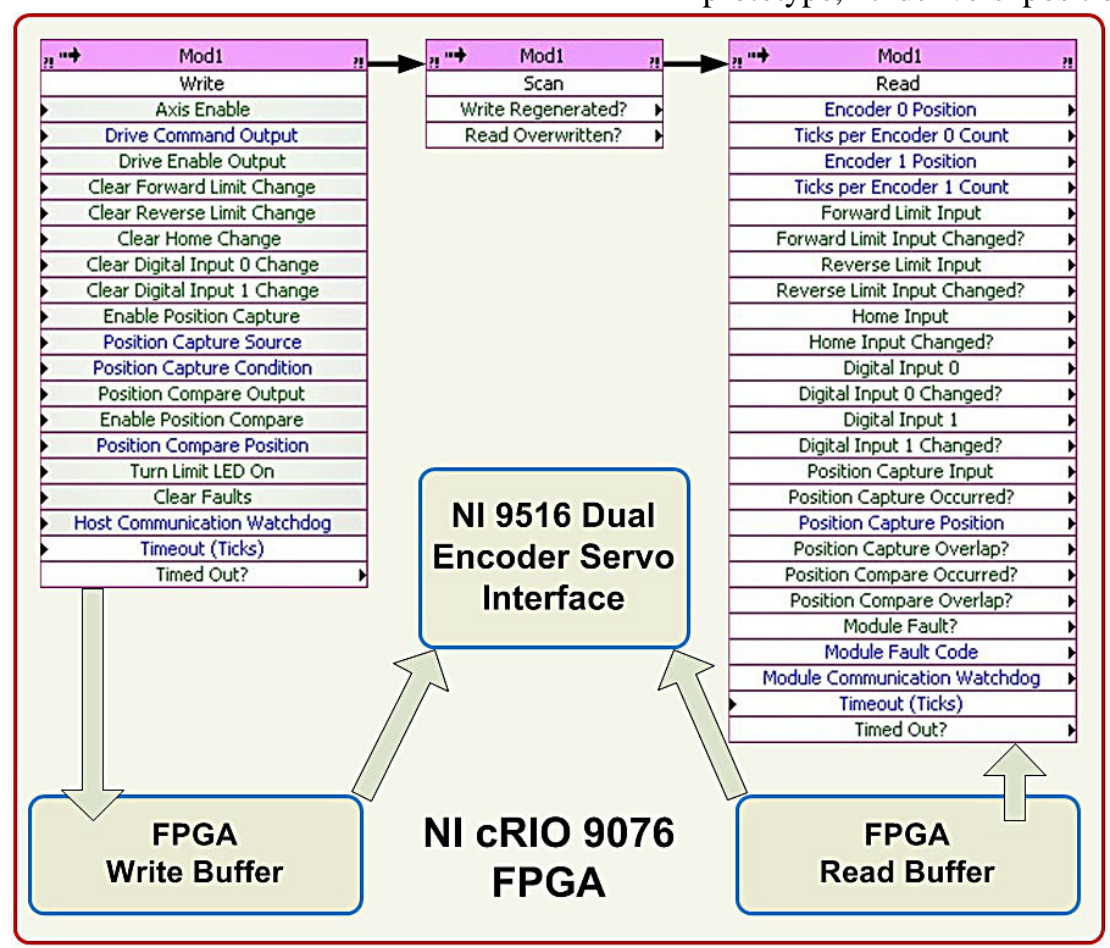

Figure 5. FPGA - NI 9516 Data Transfer Methods 
the linear and angular encoders, and the status of the end-limit proximity switches.

\section{E. FPGA Target Application}

The high-end Xilinx Spartan-6 LX45 FPGA (with 43661 logic cells, 58 multipliers and 2088Kb RAM) provides speed and flexibility of programing with the NI LabVIEW FPGA module. In motion control systems, its main application could be the execution of highly computationally-intensive complex control algorithms, while the RT host application is executing the GUI, initialisation and configuration tasks.

In this project, a PID regulator has been employed in the FPGA code - Figure 6. The main objective is to compare the speed and the functionality of this DAQ control approach with another two - the NI SoftMotion module/cRIO controller and the LabVIEW Windows/NI USB data-acquisition board.

The FPGA application has the following functions:

- Initialisation, control and signal acquisition/ monitoring of the NI 9516 servo interface module by means of provided functionality of FPGA Read/Write buffers, Write/Scan/Read methods and invoke nodes;

- Data transferring to the host application of the acquitted signals by means of DMA channel highspeed data transfer and FIFO tables;

- Generation of the custom arbitrary waveforms (excitation signals) by the use of FPGA memory blocks and Read memory method;

- Initialisation and execution of a high loop-rate PID algorithm for control of the experimental workbench;

- Exchange of various variables with the host application needed for proper functioning of the system;

- $\quad$ Adjustment and actual measurement of the FPGA loop timing according to the host requirements.

The control experiments have been performed with $100 \mu$ s loop rate timing for easier calculations and less FPGA memory consumption, but the lowest achieved loop-rate has been $33 \mu$ s even if the NI 9516 module has a guaranteed scan rate of $40 \mu \mathrm{s}$.

The main difficulties, experienced during the FPGA programming process, have been associated with the long compilation times ( 20min) and some compilation tool Xilinx 12 error messages which required the restart of the

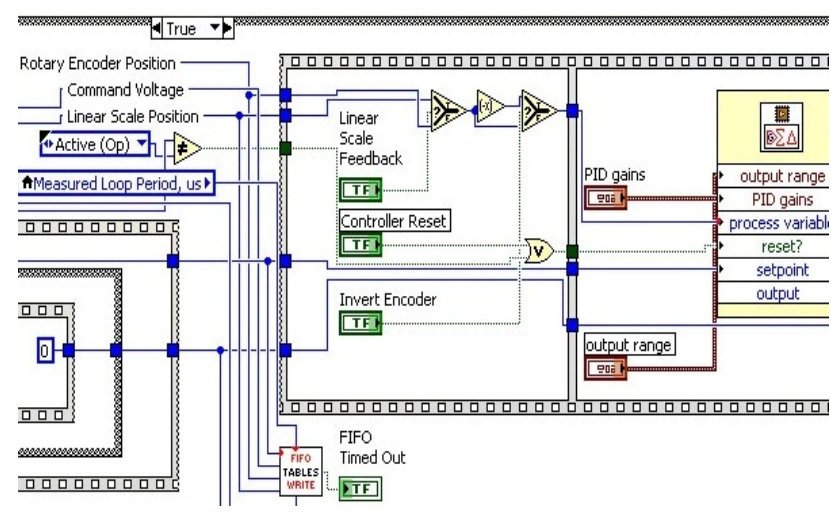

Figure 6. FPGA Target Application - Control and Signal Acquisition

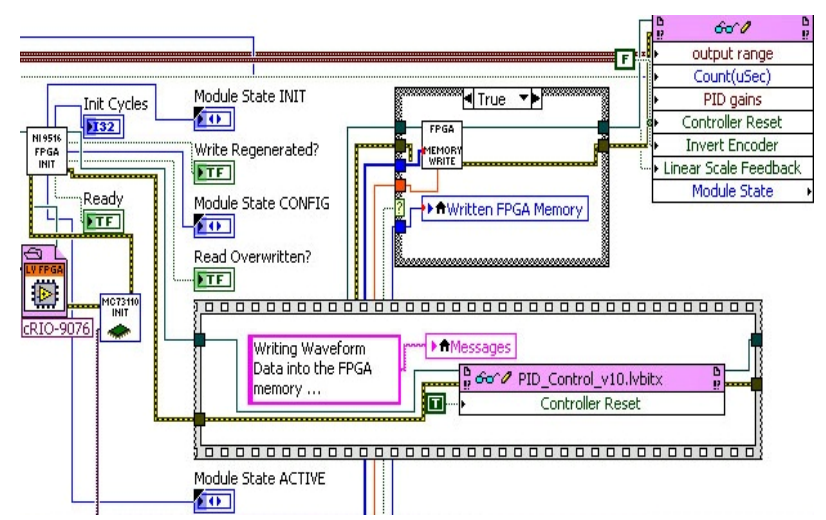

Figure 7. Host Application - Initialisation Procedures

compiler and changing the compilation strategy. However, in some cases the use of NI FPGA simulation environment could significantly reduce the prototyping times.

\section{F. Host Application}

This is the main application running within the hard RT OS. A part of the LabVIEW code containing some of the initialisation procedures is depicted in Figure 7.

The main functions of the host application could be associated with:

- Running a remote user interface and functions for the associated application and web servers;

- Implementation of a reference to the FPGA target application by means of embedded Bitfile;

- Initialisation/monitoring of the MC73110 servo controller. Separate sub-VIs have been created, namely: Initialisation, MotorON and MotorOFF;

- Initialisation of the NI 9516 servo drive interface in conjunction with the FPGA target application. A separate sub-VI has been created for configuration of the following signals:

-Drive Enable output - type and safe state;

-Linear and Angular encoder signals - phases A,B and $\mathrm{Z}$ active states and filtration;

-Home and End limits inputs signals - active states and filtration;

-Position Capture and Compare signals.

- Initialisation of the FPGA memory in conjunction with the FPGA target application. A separate subVI has been created for initialisation three data arrays with 10000 16-bit integer elements. As mentioned before, they containing the time stamps and moment amplitudes of the excitation signals. These arrays are used by the host application to initialise a specially created FPGA Block Memory domain with the same number of elements;

- Initialisation of the FPGA Target Application using Write Control Function for:

-PID parameters (Kp, Ki and Kd gains);

-FPGA loop time;

-Linear or Angular position feedback used by the PID regulator;

-Invert direction of encoder counting.

- $\quad$ Error monitoring and messaging. 
Running timed loops with different priorities in the cRIO hard real-time OS guarantees the determinism and exact timing of the running processes. On the other side, the complex algorithms are computed by the FPGA application. Thus a robust performance can be achieved.

\section{G. The LabVIEW Front Panel Interface}

The LabVIEW programming environment provides all the tools for building of a multipurpose graphical user interface - Figure 8. Several areas could be distinguished:

FPGA Current State area - provides real time information about:

- NI 9516 module operating state - Active, Initialisation, Configuration and Fault;

- $\quad$ Error codes and messages;

- Row position data - Linear scale and Resolver;

- Current address of the FPGA memory reference;

- Indicators for: NI 9516 module running, FPGA memory maximum address reached (generation of the arbitrary waveform finished) and FIFO table timed out alarm;

NI 9516 Module Faults and Errors area - provides the error codes and messages, and information for the following faults (manufacturer status register):

- Undervoltage/Overvoltage of the external power DC supply (Vsup) to the module;

- Internal power supply failure;

- $\quad$ Short Circuit of the Drive Enable output;

- Timing Synchronisation RT system problems;

- Interrupted Heartbeat (chassis) Communication;
- Module internal processor Watchdog alarm.

NI 9516 Module Initialisation area - this area is only for diagnostics. It gives the actual state of the module during the initialisation, configuration and activation stages.

Control Experiments Settings area - provides tools for setting-up of the control experiment:

- Excitation signals (arbitrary waveforms) - Square pulse, PRBS and Swept Sine;

- Waveform amplitude - the set-point to the PID regulator, expressed in $\mathrm{cm}$ of linear stage displacement;

- $\quad$ PID control parameters - Proportional gain Kc, Integral time $\mathbf{T i}$ and Derivative time $\mathbf{T d}$;

- Command voltage output range - the positive and negative limits of the output voltage;

- 16-bit scaled PID gains - since the FPGA module uses only fixed-point numbers, some conversions are needed in order the PID regulator to work properly.

Waveform Graph Window - displays the graphical data from the current experiment, expressed as:

- Excitation - in cm of linear displacement;

- $\quad$ Torque - in volts on the torque input;

- $\quad$ Linear Position - in cm of linear displacement;

- Rotary position - converted in in $\mathrm{cm}$ of linear displacement for the purposes of analysis;

- $\quad$ FPGA loop timing - in $\mu$ s of the loop period.

The aim of this experimental workbench is to provide a distant learner with a broad range of experiments in circuit

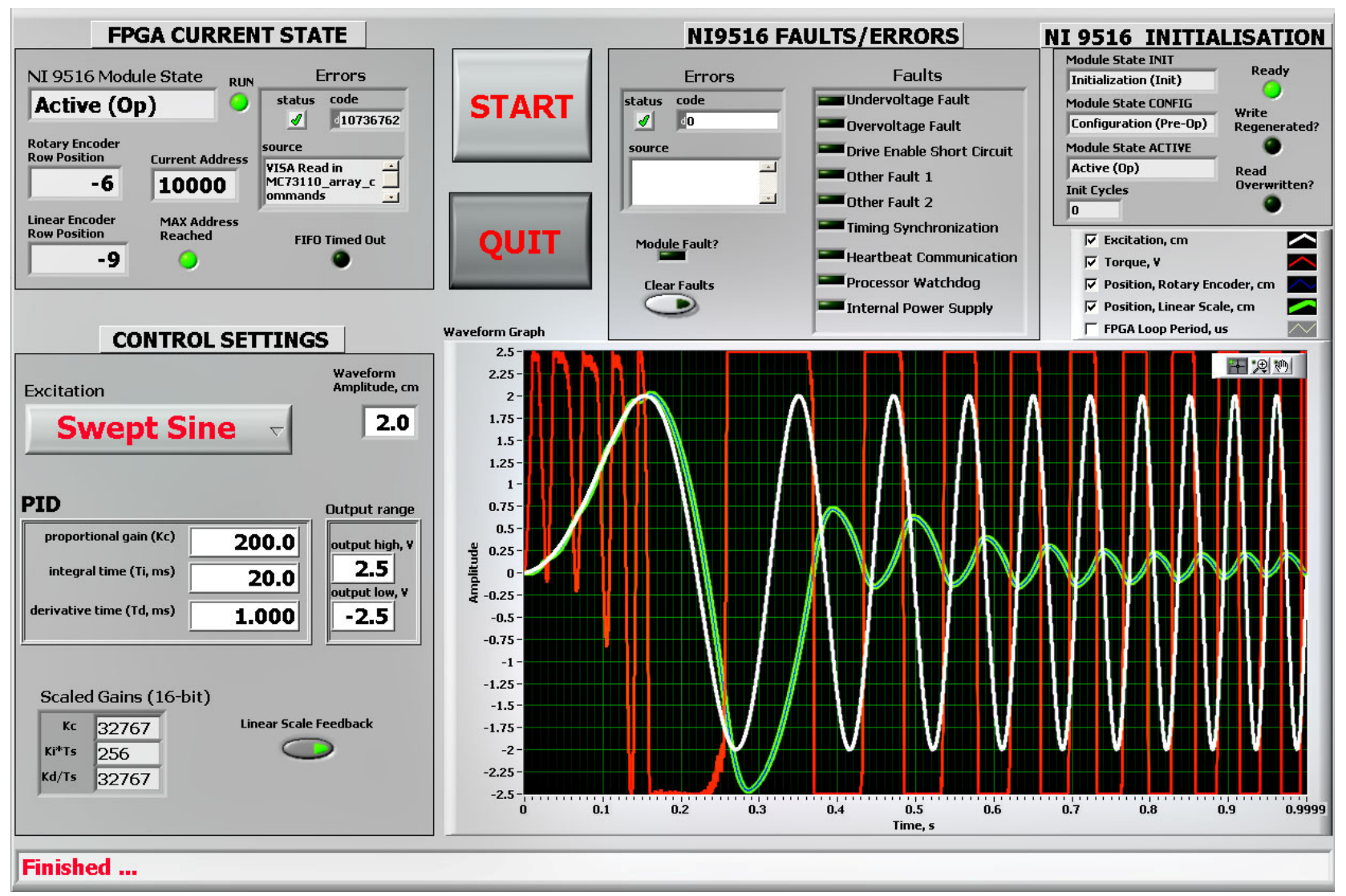

Figure 8. LabVIEW User Interface for Control of the Linear Stage in a FPGA Programming Mode 
theory, power electronics, mechatronics, motion control, feedback devices and control systems engineering. The FPGA control approach could be distinguished with its robustness, reliability and flexibility, and also with high control loop and signal acquisition speeds.

\section{OTHER DAQ CONTROL APPROACHES}

\section{A. A Control Application of NI SoftMotion Module}

As already described in [13] the NI SoftMotion module provides a variety of tools for servo axis configuration, control loop gains adjustment, testing and debugging of the whole motion system and advanced functional blocks for programming and analysing of the experiment.

Axis Configuration - the Axis Configuration window (Figure 9) is a module specific comprehensive tool for setting up a complete motion system with adjustments for:

- $\quad$ Single/Dual Loop Configurable Feedback.

- Loop Gains Configuration -Position Loop PID and Velocity Feedback parameters, Velocity and Acceleration Feed-forward parameters;

- Position and Control Loop Rates;

- Second Set of PID Gains with Gain Schedule Criteria for Acceleration Profile, Cruise Profile and Reverse Direction movements;

- $\quad$ Spline Interpolation algorithms;

- Home and Limit Switches Inputs Configuration;

- Encoders and I/O Signals Configuration ;

Gain Tuning - Following the Axis Configuration, a fine-tuning of the motion control system has been performed using the Gain Tuning Panel. It allows the loop gains to be adjusted and the response and stability to be estimated. The panel has two tabs: one is for generating a step response (in terms of position displacement and torque), and the other is for creating trajectory movement. The most important information regarding the transient response includes: Settling Time; Rise Time; Peak Time and Maximum Overshoot.

Forward and Reverse Limit Switches seeking - the NI SoftMotion module provides a number of high level

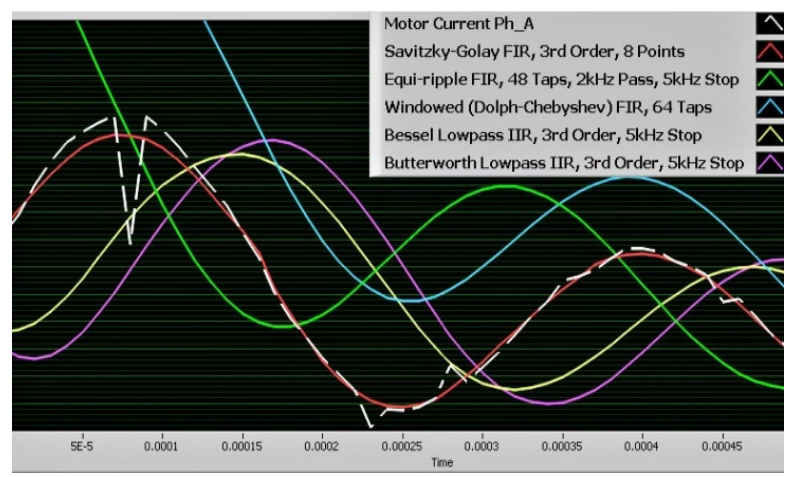

Figure 10. Motor Current Filtration with Different Filters

multi-functional blocks for movement of the controlled system and acquiring of valuable information: Find Reference Move, Straight-Line Move and Read. The needed functionality is selected by choosing the method and checking the appropriate inputs and outputs in the property window. Two timed loops synchronised with the scan engine are running with different loop rates and priorities:

Low priority loop - contains the functional blocks, which execute the reference movements and then put the linear stage in zero position. It is slower and is executed at the rate of every 10 samples of the scan engine.

High priority loop - contains the functional blocks, which acquire the real-time data for position, torque, velocity and position error during the movements.

Excitation Signals - there are a number of excitation signals which allow the moving table to be kept around at certain start point and protect the mechanics from destruction. Similar to the FPGA module, the utilised signals are: Square pulse, $P R B S$ and Swept sine.

\section{B. A Control Application of NI USB DAQ and LabVIEW}

An experimentation with the motion control workbench with NI USB-6341 DAQ running under LabVIEW for Windows has been presented in [9]. It has all the functionality of the other two control applications but

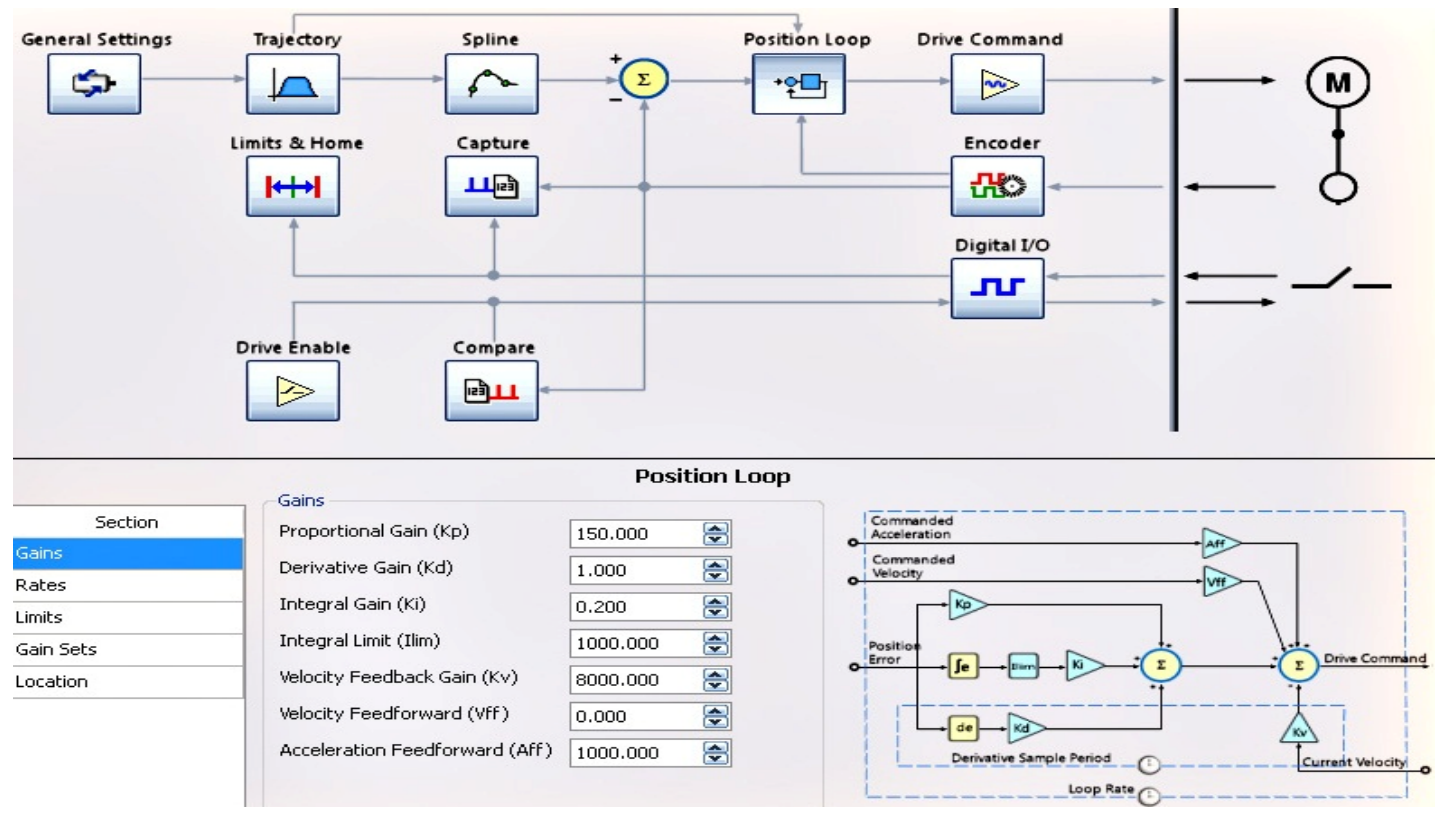

Figure 9. Axis Configuration Window for NI 9516 Servo Controller 
lacks the reliability, determinism and high control loop speed. However, it is capable of advanced data acquisition and signal processing. Figure 10 depicts the captured with $100 \mathrm{kS} / \mathrm{s}$ waveform of the motor current and its filtration with a number of FIR and IIR filters.

\section{CONCLUSIONS AND FUTURE WORK}

The presented flexible mechanical platform with realtime controller NI cRIO-9076 and NI 9516 servo interface module replicates numerous industrial applications for precise position control in the fields of micro-electronics, robotics, automotive and laser cutting industry, CNC milling machines, hard drives manufacturing, etc. The workbench is a valuable tool for remote experimentations with BLDC/PMSM motors and drives, single/multiple axes flexible mechanical systems with friction/backlash uncertainties, etc. A number of control strategies can be applied to this very specific dynamical system and their advantages and disadvantages tested and proved.

Three approaches for data acquisition and control have been developed and tested. The results are presented in Table 1 and could be summarised as:

NI cRIO with FPGA module - this is the most balanced solution in terms of PID and advanced algorithms control loop speeds, simultaneous signal acquisition sampling rates, reliability and flexibility. A significant advantage of the presented approach in the development of a motion control system is that the FPGA Target application is running various time-consuming algorithms independently from the real-time system. The development time could be high but with the use of NI simulation environment and smart programming of scalable subroutines it could be significantly reduced.

NI cRIO with SoftMotion module - this is the best solution for a robust stand-alone industrial PID control system which provides high-level programming blocks and a very short development time. It also provides a sophisticated graphical user interface that can be utilised in teaching of control system theory starting from simple experiments and moving towards more complex theory and experiments in motion control. However, the application of advanced computationally-intensive control algorithms in the RT target is practically impossible and the signal acquisition sampling rates are very low.

NI USB-6341 DAQ with LabVIEW for Windows while this approach is not suitable for robust control applications, it is highly flexible and could be a platform

TABLE I. COMPARISON OF THE TESTED APPLICATIONS

\begin{tabular}{|l|c|c|c|}
\hline & $\begin{array}{c}\text { NI DAQ } \\
\text { USB-9341 }\end{array}$ & $\begin{array}{c}\text { NI cRIO } \\
\text { SoftMotion }\end{array}$ & $\begin{array}{c}\text { NI cRIO } \\
\text { FPGA }\end{array}$ \\
\hline PID loop-rate [ms] & $70-100$ & 0.05 & $0.035-0.05$ \\
\hline $\begin{array}{l}\text { Advanced algorithms loop- } \\
\text { rate [ms] }\end{array}$ & $\begin{array}{c}\text { WINDOWS } \\
70-100\end{array}$ & $\begin{array}{c}\text { RT OS } \\
4000\end{array}$ & $\begin{array}{c}\text { FPGA } \\
0.05-0.2\end{array}$ \\
\hline $\begin{array}{l}\text { Five-signal simultaneous } \\
\text { acquisition sampling rate } \\
\text { [kS/s] }\end{array}$ & 100 & $0.1-0.5$ & $20-30$ \\
\hline Flexibility & VERY HIGH & LIMITED & HIGH \\
\hline Robustness & LOW & HIGH & HIGH \\
\hline Development time & HIGH & LOW & HIGH \\
\hline
\end{tabular}

for remote experimentation and testing different control strategies. Moreover, the NI USB-6341 is an advanced DAQ superior to other solutions for signal acquisition. That makes it particularly useful for system identification and model validation purposes.

\section{REFERENCES}

[1] J. S. Falcon and M. Trimborn, "Graphical programming for field programmable gate arrays: applications in control and mechatronics," in American Control Conference, 2006, pp. 13941400 .

[2] J. Sedillo and J. Gilpatrick, "RIO-based wire scanner motion control," in PAC09, Vacouver, BC, Canada, 2012, pp. 3624-3626.

[3] N. Hoshi, Y. Utsumi, and K. Oguchi, "A Web-accessible FPGAbased Direct Torque Controller for Permanent Magnet Synchronous Motor," in 4th International Power Electronics and Motion Control Conference, IPEMC'04, Xi 'an, China, 2004, pp. 1325-1330.

[4] A. Rojko, D. Hercog, and K. Jezernik, "Power Engineering and Motion Control Web Laboratory: Design, Implementation and Evaluation of Mechatronics Course," IEEE Transactions on Industrial Electronics, vol. PP, pp. 1-1, 2009.

[5] (2012, 30 ${ }^{\text {th }}$ of August). NI cRIO-9076 Integrated $400 \mathrm{MHz}$ RealTime Controller and LX45 FPGA. Available: http://sine.ni.com/nips/cds/view/p/lang/en/nid/209758

[6] (2012, 30 ${ }^{\text {th }}$ of August). NI 9516 C Series Servo Drive Interface with Dual Encoder Feedback. Available: http://sine.ni.com/nips/ cds/view/p/lang/en/nid/206349

[7] (2012, $12^{\text {th }}$ of October). LabVIEW NI SoftMotion Module. Available: http://sine.ni.com/nips/cds/view/p/lang/en/nid/14234

[8] (2013, 20 $0^{\text {th }}$ of February). NI LabVIEW FPGA Module. Available: http://sine.ni.com/nips/cds/view/p/lang/en/nid/11834

[9] S. D. Gadzhanov, A. Nafalski, and Z. Nedic, "A Universal Workbench for Motion Control Experimentations in LabVIEW Environment," in $9^{\text {th }}$ International Conference on Remote Engineering and Virtual Instrumentation (REV), Bilbao, Spain, 2012, pp. 51-57. http://dx.doi.org/10.1109/REV.2012.6293106

[10] (2010, 21 $1^{\text {st }}$ of May). MC73110 For Brushless DC Motor Control. Available: http://www.pmdcorp.com/advanced-motion-control/ brushless-dc-motor-controller.cfm

[11] (2010, 21 ${ }^{\text {st }}$ of May). AD2S1210: Variable Resolution, 10-Bit to 16-Bit $R / D$ Converter with Reference Oscillator. Available: http://www.analog.com/en/analog-to-digital-converters/synchro resolver-to-digital-converters/AD2S1210/products/product.html

[12] (2012, 29 $9^{\text {th }}$ of February). NI USB-6341 X-Series Data Acquisition. Available: http://sine.ni.com/nips/cds/view/p/lang/en/ nid/209069

[13] S. D. Gadzhanov, A. Nafalski, and Z. Nedic, "An Application of NI SoftMotion RT System in a Motion Control Workbench," in $10^{\text {th }}$ International Conference on Remote Engineering and Virtual Instrumentation (REV), Sydney, Australia, 2013.

\section{AUTHORS}

S. D. Gadzhanov is with the School of Electrical and Information Engineering, University of South Australia, Mawson Lakes, Australia (e-mail: Stamen.Gadzhanov@unisa.edu.au).

A. Nafalski is with the School of Electrical and Information Engineering, University of South Australia, Mawson Lakes, Australia (e-mail: Andrew.Nafalski@ unisa.edu.au).

Z. Nedic is with the School of Electrical and Information Engineering, University of South Australia, Mawson Lakes, Australia (e-mail: Zorica.Nedic@ unisa.edu.au).

This article is an extended and modified version of a paper presented at the International Conference on Remote Engineering \& Virtual Instrumentation (REV2012), held at University of Deusto, Bilbao, Spain, July 4-6, 2012. Received 01 March 2013. Published as resubmitted by the authors 20 March 2013. 